\title{
The Role of Attentional Control and Resilience in Predicting the Rumination in Patients With Obsessive-Compulsive Disorder
}

\author{
Samaneh Behzadpoor, ${ }^{1,{ }^{*}}$ Faramarz Sohrabi, ${ }^{2}$ and Ahmad Borjali ${ }^{2}$ \\ ${ }^{1}$ Department of Psychology, Faculty of Education and Psychology, Shahid Beheshti University, Tehran, IR Iran \\ ${ }^{2}$ Department of Psychology, Faculty of Psychology and Education, Allameh Tabatabaei University, Tehran, IR Iran \\ "Corresponding author: Samaneh Behzadpoor, Department of Psychology, Faculty of Education and Psychology, Shahid Beheshti University, Tehran, IR Iran. E-mail: \\ samaneh_behzadpoor@yahoo.com
}

Received 2015 May 28; Accepted 2015 November 17.

\begin{abstract}
Background: Rumination is one of the extreme negative cognitions in patients with obsessive compulsive disorder (OCD). It is assumed that some cognitive problems such as attentional control can facilitate rumination and some internal capabilities such as resiliency can reduce engaging in these ruminations.

Objectives: The present study investigated the relationship between rumination with attentional control and resiliency in patients with OCD.

Patients and Methods: In this correlational study, 50 patients with OCD were selected through accessible purposive sampling. The data was obtained through ruminative response, Connor and Davidson resiliency, and scalability questionnaires and were analyzed using the Pearson correlation and multiple regression tests.

Results: The results indicated a significant negative correlation $(P=0.001)$ between rumination and attentional control $(r=0.573)$ and resiliency $(r=0.579)$. Results of regression analysis indicated increased attentional control and resiliency can predict reduction of rumination $(\mathrm{P}=0.001)$.

Conclusions: Regarding that attentional control and resiliency are associated with rumination and can predict rumination in patients with obsession, nurturing resiliency traits and teaching techniques to improve attentional control can be effective in the control and prevention of rumination.
\end{abstract}

Keywords: Rumination, Attention, Resilience, Obsessive Compulsive Disorder

\section{Background}

Among anxiety disorders, a disorder that is defined on the basis of additional negative cognitions, is obsessive compulsive disorder(OCD)[1]. Rumination which includes excessive and negative emotional cognitions, is one of the cognitive characteristics of these patients $[1,2]$. Rumination is defined as persistent and recurrent thoughts that turns around a common theme [3]. Rumination separates the person from his environment and focuses him on the inner thoughts [4].

Rumination in OCD patients includes thoughts that are reviewed inexhaustibly and lead to hopelessness [5]. Several studies have specifically examined the rumination in obsession, all of which have reported increase of rumination in patients with obsession $[2,6,7]$. When negative thoughts enter the mind, on the one hand, the mind thinks of individual inadequacy, unrealistic goals, interpersonal concerns and reminding unenjoyable subjects. On the other hand, he is worried to lose the valuable information that can help him to solve the problems. Fortu- nately, most people tend to solve this dilemma through the efficient attention to negative thoughts, but some people are incapable of achieving this kind of mental control and use repression and rumination [8]. Loss of mental control is one of the key complaints of psychological disorders and specifically OCD. One of the components of cognitive control and emotional control is attentional control [9]. Attentional control means the monitoring of all components of attention and is necessary to adjust flexibility of cognitive resources $[10,11]$.

In fact, the ability of cognitive control, including attentional is considered as a vital information processing factor which is associated with the tendency to rumination $[12,13]$. Researches in this field have shown that deficiency in cognitive control, including deficiency in attentional control, predicts the tendency to rumination $[14,15]$.

On the other hand, some researchers describe rumination as cognitive focus on negative emotions [16, 17]. In other words, rumination can be caused by the inability in managing emotions. An acquired trait which increases a person's ability to manage emotions is resiliency 
[18]. Resiliency can possibly reduce engagement in rumination [19]. Mohammadkhani et al. [20, 21] indicated that increased rumination is associated with low levels of resiliency [20]. Results of the studies conducted by Togad and Fredrickson and also by George indicated that resiliency is negatively associated with rumination.

OCD is confronting research problems in which cognitive problem and the interaction of cognitive deficits are investigated in these individuals, because OCD is primarily a cognitive processing disorder [9]. If in fact, the deficiency in attention affects rumination, the treatments that focus on the cognitive impairment, can help reduce the patient's obsessive thoughts and rumination and can also increase a person's ability to control thoughts. On the other hand, if abilities such as resiliency can help prevent the onset of symptoms such as rumination, teaching its components to individuals with obsession can be helpful. On the other hand, less research has directly examined rumination in patients with OCD and its predictors.

\section{Objectives}

Therefore, the purpose of this research is to predict rumination based on the attentional control and resiliency in patients with OCD.

\section{Patients and Methods}

In this descriptive-correlational study, population consisted of all patients who suffer from OCD who referred to health centers in Tehran province in 2012 (April to September). Using the accessible purposive sampling method, 50 individuals were diagnosed with OCD and were selected after the interview by the psychiatrist and based on DSM-IV criteria. The enter criteria include: having OCD diagnostic criteria according to the psychiatrist and based on clinical interview, having minimum 18 years of age and maximum 60 years, having a minimum education level of third grade degree at junior high school, the patients consent to participate in the study. The exclusion criteria include: having psychotic symptoms and drug abuse, having full criteria of other psychological disorders on axis I and having the full criteria for the personality disorders on axis II according to the psychiatrist's diagnosis based on the diagnostic interview. The subjects responded self-report questionnaires of rumination, attention control, and resilience after the examination and diagnosis by a psychiatrist, having the desired criteria and establishing a good relationship with the researcher.

The questionnaires that we used for gathering data include:
1) Attention control scale (ACS): The ACS is a 20-item self-report questionnaire that assesses control of attention across two domains; focusing, the ability to maintain attention on a given task, and shifting, the ability to reallocate attention to a new task or to engage attention on multiple tasks. Each item is rated on a 4-point Likert scale from " 1 " (almost never) to " 4 " (always) with higher scores indicative of better attentional control. The ACS total score had adequate internal consistency $(\alpha=0.86)$, as did the focus and shifting subscales ( $\alpha=0.82$ and $\alpha=0.75$, respectively), in the present sample. It is important to note that the ACS does not contain questions related to emotionallyvalenced situations, and as such attempts to capture a general information processing trait uncontaminated by reactions to emotional stimuli or cognitions [6]. This questionnaire was translated into Persian by the researcher; first, the items of the questionnaire were translated into Persian by the researcher and a MA in English language. Then with using the back translation by another translator with MA degree in English, the accuracy of the translation was approved. Ultimately, the final version was prepared according the ideas of psychology professors. The face and content validity was confirmed by the psychology professors. Cronbach's alpha coefficient was obtained as 0.87 in patients with OCD.

2) Ruminative response test (RRS): The RRS consists of 22 items measuring ruminative responses. In previous studies, total RRS has achieved a test-retest correlation of 67 over a 2-year period and satisfactory convergent and predictive validity [22]. Reliability of the RRS was examined by assessing internal consistency coefficient (Cronbach alpha). Cronbach's alpha were found for reflection subscale $\alpha=0.58$ and brooding subscale $\alpha=0.58$ and total score of short RRS $\alpha=0.72$. Convergent validity of the short version of RRS was examined by computing Pearson correlation coefficients with the brief symptom inventory (BSI) scale. In current study there were significantly high positive correlation between short RRS and BSI scores $(r=0.59, \mathrm{P}=0.01$ ). Furthermore, there was significant positive correlation between reflection subscale and BSI scores $(\mathrm{r}=0.44, \mathrm{P}=0.01)$ and between brooding subscale and BSI scores $(\mathrm{r}=0.52, \mathrm{P}$ $=0.01)$ [23].

3) Connor and Davidson Resilience Scale (CDRISC): The CD-RISC was developed by Connor and Davidson. It is composed of 25 items on a five-point Likert scale ranging from 'not true at all' to 'true nearly all the time'. The CD-RISC measures the ability to cope with stress and threat. Connor and Davidson reported the adequate reliability and validity of this measure in different groups (normal and prone to danger populations) [19]. In research of Fu, Leoutsakos \& Carol the internal consistency of the total CDRISC was 0.86 . Internal convergent validity was examined 
based on the strength of correlations between items of the CD-RISC scale. Polychoric correlations showed positive correlations between items, 0.17 and 0.69 . Items related to PTSD loaded on one factor while items related to resilience loaded on dissimilar factors demonstrating discriminant construct validity. The scale exhibited external construct validity with high resilience scores had statistically significantly lower depression scores [24].

Data analyses were also done through interpretive statistics (mean and standard deviation) and Pearson correlation and multiple regression tests. SPSS-18 software was used to analyze data.

\section{Results}

The mean age of participants was 35.3 in the range of 18 to 53 . Participants were $40 \%$ male and $60 \%$ female. Also they were $54 \%$ single and $46 \%$ married. Regarding education $6.7 \%$ had high school studies, $46.7 \%$ had diploma, 23.3\% had associate degree and $23.3 \%$ had bachelor's degree.

The mean and standard deviation scores of variables are provided in table 1 . Pearson's correlation coefficient between the variables is provided in table 1 . There is a significant positive correlation between rumination and attentional control $(r=0.59)$ and resiliency $(r=0.579)$ and these coefficients are significant at the level of 0.01 (Table 1 ).

In order to investigate the significance of the regression model, one-way ANOVA was used. According to the results in Table 2 and the amount of the observed $F$, the regression of rumination variable on the control variables and resilience is significant at the level of 0.01. In other words, these variables explain a part of the rumination variance (Table 2 ).

The summary of the statistics of regression model are provided in Table 2 . According to this table and $\chi^{2}$ correlation index $\left(\mathrm{R}^{2}=0.542\right)$, we can conclude that the predictor variables (attentional control and resilience) explain 54.2\% of rumination (Table 2 ).

Also as can be seen in Table 2 and with respect to the Beta value, resilience $($ Beta $=0.475)$ and attentional control $($ Beta $=0.466)$ respectively predict rumination in reverse at the significance level of 0.01 (Table 2).

\section{Discussion}

Based on research findings, there is a negative significant relationship between rumination and attentional control. Also according to the results of the regression analysis, we can say that control attention can negatively predict rumination. In other words, the more deficiency an in individual has in attentional control, the probability of more rumination increases. A look at past research suggests that this finding is consistent with the results of Koster et al., Gotlib and Joormann, De Lissnyder et al. [1214]. All these studies suggest that the deficiency in attentional control and cognitive control can lead to rumination. Watkins and Brown suggest that rumination reduces cognitive flexibility by overloading cognitive resources, primarily in the form of deficits in switching and inhibition [25]. Whitmer and Banich [26] suggest that switching attention from one conceptual set to another is difficult for individuals who ruminate. In the explanation of this finding, we can say that rumination can be facilitated due to deficiency in focus of attention so that when the focus of attention is in trouble, the unwanted negative thoughts would enter the memory despite the efforts of suppressing them. The disability of a person to prevent the engagement of attention with these obsessive thoughts and rumination may increase due to a deficiency in the displacement of attention. Focus of attention and displacement are two aspects of attentional control. Therefore, the loss of attentional control can increase rumination and can facilitate it [6].

On the other hand, the results suggested that resilience has a negative relationship with rumination and resilience can significantly and negatively predict rumination. The results of this study confirm the findings of a Mohammadkhani et al., Tugade and Fredrickson and George $[20,21,27]$. All these studies indicated that resilience has a negative relationship with rumination. In fact, the resilience attributes acts as a source of enduring source and prevents more severe symptoms in patients with OCD. Enduring individuals have more power in managing their emotions [19] and solving problems [28]. Resilient people, regarding the location, are aware and know their feelings and emotions, and are also aware of the causes of these feelings and manage their emotions in healthy ways [19]. This helps enduring people to cope with these conditions well in case of illness or stress and manage their emotions in a healthy way. Also the ability of problem-solving in enduring individuals enables the individual to apply efficient ways to improve his illness symptoms rather than passively engaging in intellectual. Rumination has a host of negative impacts on us physically and mentally. Ruminators have chronically elevated levels of the fight-orflight hormones adrenaline and cortisol in response to the anxiety they are conjuring, making them constantly overactivated physically and emotionally. Resilience lies in the ability to reflect on pressures without generating the debilitating physical and mental effects of stress, making people fit to tackle the pressures. The key is eliminating the negative emotional aspects associated with rumination. It is the negative emotional aspects that put people physically and emotionally into fight-or-flight hormonal over- 
Table 1. Descriptive Statistics of the Study Variables and Correlation Coefficients of the Studied Variables

\begin{tabular}{|c|c|c|c|}
\hline Variables & Descriptive Statistics of Variables (Mean \pm SD) & Correlation Coefficients of the Studied Variables & P Value \\
\hline Rumination & $62.38 \pm 13.14$ & & \\
\hline Attentional control & $45.94 \pm 8.97$ & 0.59 & 0.001 \\
\hline Resiliency & $48.74 \pm 11.71$ & 0.579 & 0.001 \\
\hline
\end{tabular}

Table 2. Indexes of One-Way ANOVA to Evaluate the Significance of the Regression Model and Summary Statistics of the Regression Model

\begin{tabular}{|c|c|c|c|c|c|c|c|c|c|}
\hline Value & Sum of Squares & Degree of Freedom & The Mean Sum of Squares & $\mathbf{F}$ & PValue & Correlation $(\mathrm{R})$ & Squared Correlation $\left(\mathrm{R}^{2}\right)$ & Adjusted Square Correlation & Estimated SD \\
\hline Regression & 4588.182 & 2 & 2294.091 & 27.806 & 0.001 & 0.736 & & & \\
\hline Remaining & 3877.598 & 47 & 82.502 & & & & 0.542 & 0.532 & 0.084 \\
\hline Total & 8465.780 & 49 & & & & & & & \\
\hline
\end{tabular}

drive, and that ruminators marinate in [29].

The major limitation of the research was related to the students' community whose results can be cautiously generalized to other communities. Research method (correlation) also puts limitations regarding the interpretations and etiological predications of variables under study. This must be considered too. Finally, as it was found in this study, the characteristic of resilience and as higher attentional control in patients with OCD can reduce rumination and somehow prevent its occurrence, it is recommended to control and prevent rumination through teaching techniques such as cognitive techniques for controlling attention and teaching resilience parameters to people with obsession.

To meet the ethics and increase participation, participants were informed about the subject and objective of the study to the extent that does not affect the results. After reassuring participants regarding the matter that they would never examined individually and can leave the research anytime, they entered into the study. It must be noted that participants were not asked for their names.

\section{Acknowledgments}

Hereby we thank officials of Imam Hussein (AS) hospital and Dr. Shams who had cooperated fully in this investigation.

\section{Footnotes}

Authors' Contribution: All authors had equal role in design, work, statistical analysis and manuscript writing.

Funding/Support: This paper had been done by personal expenses.

\section{References}

1. Salmani B, Hasani J. Cognitive attentional syndrome (cas) \& cognitive emotion regulation strategies: Transdiagnostic processes or diagnostic based on mood \& anxiety disorders [in Persian].J Clin Psychol. 2013;5(3):91-104.

2. Sarraj-Khorrami A, Karami J, Momeni KM. Comparing thinking rumination and defense mechanisms in patients suffering from major depression and obsessive- compulsive disorders with normal individuals [in Persian].J Clin Psychol. 2014;6(2):53-63.

3. Joormann J. Differential effects of rumination and dysphoria on the inhibition of irrelevant emotional material: Evidence from a negative priming task. Cognit Ther Res. 2006;30(2):149-60. doi:10.1007/s10608006-9035-8.

4. Martell CR, Dimidjian S, Herman-Dunn R. Behavioral activation for depression. New York: Guilford Press; 2013.

5. Clark DA. Cognitive-behavioral therapy for OCD. 1 ed. New York: Guilford Press; 2006.

6. Armstrong T, Zald DH, Olatunji BO. Attentional control in OCD and GAD: specificity and associations with core cognitive symptoms. Behav Res Ther. 2011;49(11):756-62. doi:10.1016/j.brat.2011.08.003. [PubMed: 21871608].

7. Grisham JR, Williams AD. Cognitive control of obsessional thoughts. Behav Res Ther. 2009;47(5):395-402. doi: 10.1016/j.brat.2009.01.014. [PubMed: 19232410].

8. Papajerjeyou C, Welz A. Depressive rumination: Nature, theory, and treatment. 1 ed. New York: Wiley Press; 2003.

9. Shams G, Sadeghi M. Recent advances in cognitive aspects of obsessive-compulsive disorder. Adv Cogn Sci. 2010;12(1):1-16.

10. Derryberry D, Reed MA. Anxiety-related attentional biases and their regulation by attentional control. J Abnorm Psychol. 2002;111(2):22536. [PubMed: 12003445].

11. Posner MI, Rothbart MK. Research on attention networks as a model for the integration of psychological science. Annu Rev Psychol. 2007;58:1-23. doi: 10.1146/annurev.psych.58.110405.085516. [PubMed: 17029565].

12. Koster EH, De Lissnyder E, Derakshan N, De Raedt R. Understanding depressive rumination from a cognitive science perspective: the impaired disengagement hypothesis. Clin Psychol Rev. 2011;31(1):138-45. doi: 10.1016/j.cpr.2010.08.005. [PubMed: 20817334].

13. Gotlib IH, Joormann J. Cognition and depression: current status and future directions. Annu Rev Clin Psychol. 2010;6:285-312. doi: 10.1146/annurev.clinpsy.121208.131305. [PubMed: 20192795]. 
14. De Lissnyder E, Koster EH, Goubert L, Onraedt T, Vanderhasselt MA, De Raedt R. Cognitive control moderates the association between stress and rumination. J Behav Ther Exp Psychiatry. 2012;43(1):519-25. doi: 10.1016/j.jbtep.2011.07.004. [PubMed: 21813083].

15. Joormann J, Gotlib IH. Emotion regulation in depression: relation to cognitive inhibition. Cogn Emot. 2010;24(2):281-98. doi: 10.1080/02699930903407948. [PubMed: 20300538].

16. Michael ST, Snyder CR. Getting unstuck: the roles of hope, finding meaning, and rumination in the adjustment to bereavement among college students. Death Stud. 2005;29(5):435-58. doi 10.1080/07481180590932544. [PubMed: 15971360].

17. Morrow J, Nolen-Hoeksema S. Effects of responses to depression on the remediation of depressive affect.JPers Soc Psychol. 1990;58(3):51927. [PubMed: 2324941].

18. Lee JE, Sudom KA, Zamorski MA. Longitudinal analysis of psychological resilience and mental health in Canadian military personnel returning from overseas deployment. J Occup Health Psychol. 2013;18(3):327-37. doi:10.1037/a0033059. [PubMed: 23834447]

19. Connor KM, Davidson JR. Development of a new resilience scale: the Connor-Davidson Resilience Scale (CD-RISC). Depress Anxiety. 2003;18(2):76-82. doi:10.1002/da.10113. [PubMed:12964174].

20. Mohammadkhani S, Soleimani H, Naghei S. A. . The role of emotional schemas in resilience of people living with HIV. Knowl Health J. 2014;9(3):1-10.

21. Tugade MM, Fredrickson BL. Resilient individuals use positive emotions to bounce back from negative emotional experiences. J Pers So Psychol. 2004;86(2):320-33. doi: 10.1037/0022-3514.86.2.320. [PubMed: 14769087].
22. Butler AC, Chapman JE, Forman EM, Beck AT. The empirical status of cognitive-behavioral therapy: a review of meta-analyses. Clin Psychol Rev. 2006;26(1):17-31. doi: 10.1016/j.cpr.2005.07.003. [PubMed: 16199119].

23. Erdur-Baker O, Bugay A. The short version of ruminative response scale: reliability, validity and its relation to psychological symptoms. Procedia Soc Behav Sci. 2010;5:2178-81. doi:10.1016/j.sbspro.2010.07.433.

24. Fu C, Leoutsakos JM, Underwood C. An examination of resilience cross-culturally in child and adolescent survivors of the 2008 China earthquake using the Connor-Davidson Resilience Scale (CD-RISC). J Affect Disord. 2014;155:149-53. doi: 10.1016/j.jad.2013.10.041. [PubMed: 24215898].

25. Watkins E, Brown RG. Rumination and executive function in depression: an experimental study. J Neurol Neurosurg Psychiatry. 2002;72(3):400-2. [PubMed: 11861707].

26. Whitmer AJ, Banich MT. Inhibition versus switching deficits in different forms of rumination. Psychol Sci. 2007;18(6):546-53. doi: 10.1111/j.1467-9280.2007.01936.x. [PubMed: 17576269].

27. George K. Resilience in academic stress: Exploring the role of cognition in how students adjust to life at the University of New York. Heslington: University of New York; 2014.

28. Tainter JA, Taylor TG. Complexity, problem-solving, sustainability and resilience. Build Res Inf. 2014;42(2):168-81. doi: 10.1080/09613218.2014.850599.

29. Muir R. Managing stress: Rumination and You 2013. Available from: http://www.lawpeopleblog.com/2013/09/managing-stressrumination-and-you. 